\title{
Eficiencia in vitro de hongos entomopatógenos y productos químicos sobreRhipicephalus microplus
}

\section{ARTÍCULO DE INVESTIGACIÓN}

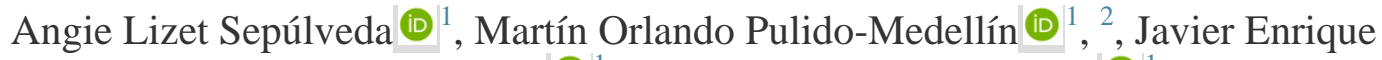 \\ Rodríguez-Pacheco (iD ${ }^{1}$, Diego José García-Corredor (iD ${ }^{1}$
1 Grupo de Investigación en Medicina Veterinaria y Zootecnia (GIDIMEVETZ), Programa de Medicina Veterinaria y Zootecnia, Universidad Pedagógica y Tecnológica de Colombia, Boyacá, Colombia.

\author{
mopm1@hotmail.com
}

Recibido: 19 de Enero de 2017 y aprobado: 11 de Abril de 2017, Actualizado: 8 de Septiembre de 2017

DOI: 10.17151/vetzo.2017.11.2.6

RESUMEN: Rhipicephalus microplus es el ectoparásito más importante en bovinos, su presencia reduce la producción, ocasiona pérdida de peso y es vector de enfermedades como anaplasmosis y babesiosis. El control de garrapatas se realiza mediante el uso de ixodicidas favoreciendo el desarrollo de garrapatas resistentes y generando residualidad, lo que sugiere la utilización de sistemas alternativos de control. Con el fin de evaluar la eficiencia in vitro de Cordyceps bassiana (Cepa BbF2011),Metharizium anisopliae (Cepa MAF1309) y productos químicos en la fase adulta de $\boldsymbol{R}$. microplus se colectaron garrapatas hembras pletóricas de bovinos, se empleó la técnica de inmersión de Drummond utilizando una dilución de $1 \times 10^{8}$ esporas$\mathrm{ml}$ para los hongos, un organofosforado (OF) al 97\%, un piretroide (PS) al 15\% y amitraz (AM) al 20,8\%. Las garrapatas tratadas con $\boldsymbol{C}$. bassiana alcanzaron el $100 \%$ de mortalidad el día 10 post-tratamiento (PT), seguida de $\boldsymbol{M}$. anisopliae y OF los cuales llegaron al 100\% de mortalidad los días 11 y 14, respectivamente. Los tratamientos con AM, PS y control no alcanzaron el $100 \%$ de la mortalidad. El efecto de $\boldsymbol{C}$. bassiana y $\boldsymbol{M}$. anisopliae sobre la fase adulta de garrapatas genera nuevas alternativas de control deR. microplus.

Palabras clave: control químico, garrapatas, hongos entomopatógenos, Rhipicephalus microplus (Decs).

\section{In vitro efficiency of entomopathogenic fungi and chemicals on Rhipicephalus microplus}

\begin{abstract}
Rhipicephalus microplus is the most important ectoparasite in cattle and its presence reduces production, causes weight loss and is a vector of diseases such as anaplasmosis and babesiosis. Ticks control is carried out by the use of ixodicides favoring the development of resistant ticks and generating residuality, which suggests the use of alternative control systems. To evaluate the in vitro efficiency of Cordyceps
\end{abstract}


bassiana (Strain BbF2011), Metharizium anisopliae (Strain MAF1309) and chemical products in the adult phase of R. microplus, female ticks were collected from bovines using the Drummond immersion technique with dilution of 1x108 spores-ml for fungi, organophosphorus (OF) to $97 \%$, pyrethroid (PS) to $15 \%$ and amitraz (AM) to $20.8 \%$. Ticks treated with $\boldsymbol{C}$. bassiana reached $100 \%$ mortality on day 10 post-treatment (PT), followed by M. anisopliae and OF, which reached 100\% mortality on days 11 and 14 respectively. The treatments with AM, PS and control did not reach $100 \%$ mortality. The effect of $\boldsymbol{C}$. bassiana and $\boldsymbol{M}$. anisopliae on the adult stage of ticks generates new alternatives for the control of R. microplus.

Key words: ticks, entomopathogenic fungi, chemicals, Rhipicephalus microplus.

\section{Introducción}

En la actualidad se reconocen más de 907 especies de garrapatas distribuidas en el mundo (Cortes-Vecino et al., 2010), muchas de ellas pueden transmitir patógenos como bacterias, helmintos, protozoos y virus que afectan al hombre y animales domésticos (Brahma et al., 2014; Galay et al., 2015). Sin embargo, la garrapata Rhipicephalus microplus, conocida como garrapata común del ganado bovino, es sin duda el ectoparásito que con mayor frecuencia parasita los ganados en las regiones en Centro, Sudamérica (González et al., 2011), Australia y parte de África (Cortes-Vecino et al., 2010). Chagas et al. (2014) coinciden con De Clercq et al. (2015) al afirmar a $\boldsymbol{R}$. microplus como una de las especies de garrapatas con mayor distribución en regiones tropicales y subtropicales del mundo afectando aproximadamente el $80 \%$ de la población bovina, encontrándose desde el nivel del mar hasta los $2903 \mathrm{msnm}$ y a temperaturas que oscilan entre 15 y $34^{\circ} \mathrm{C}$ (Cortes-Vecino et al., 2010).

Debido a los hábitos hematófagos del parasito, su presencia es considerada uno de los principales problemas de salud pública con gran impacto económico en la ganadería bovina del mundo (Rodríguez-Vivas et al., 2010). Ren et al. (2012) y Antunes et al. (2015) indican que $\boldsymbol{R}$. microplus es una de las garrapatas de mayor relevancia por ser considerada vector de enfermedades en animales, específicamente transmite babesiosis (Babesia bigemina y Babesia bovis) y anaplasmosis (Anaplasma marginale). Además, se describe cómo a partir de 20-30 garrapatas por animal se producen pérdida de peso, daños en la piel por acción de las picaduras, pérdida de sangre, debilitamiento, estrés, disminución en la producción de leche y menor eficiencia reproductiva del hato (Rodríguez-Vivas et al., 2010; Rodríguez-Vivas et al., 2012).

La presencia de este ectoparásito ocasiona gran impacto a nivel económico dentro de las explotaciones ganaderas. A nivel mundial se registran pérdidas entre 2000 a 3000 millones de dólares (FAO, 2013). Monteiro et al. (2014) coinciden en establecer a $\boldsymbol{R}$. microplus como uno de los principales obstáculos en la producción ganadera en regiones tropicales y subtropicales. Se estima que es la responsable de pérdidas económicas anuales que rondan los \$US 2,5 billones alrededor del mundo, mientras en Colombia las pérdidas ascienden a 76.713 millones de pesos por año (Cortes-Vecino et al., 2010). 
Los métodos actuales para el control de la garrapata implican el uso de métodos químicos y no químicos. Las estrategias de control de esta parasitosis incluyen el uso extensivo, y muchas veces indiscriminado, de compuestos químicos ixodicidas de las familias de los organofosforados, piretroides sintéticos, amidinas, fenilpirazolonas y lactonas macrocíclicas, los cuales muestran actualmente diferentes niveles de resistencia en el campo, además de que su uso representa un riesgo ambiental y de salud pública ampliamente reconocido (Rodríguez-Vivas et al., 2012; Cruz-Vázquez et al., 2015). Diversos estudios han comprobado que el uso de hongos entomopatógenos no solo causa la mortalidad de muchas especies de garrapatas, sino que también tiene gran eficacia sobre su reproducción (Fernández et al., 2010; Pulido-Medellín et al., 2015; García-Corredor et al., 2016), constituyéndose como una de las alternativas no químicas para su control (Cardona et al., 2005; Ortiz-Urquiza et al., 2015).

Se ha demostrado la eficacia de $\boldsymbol{M}$. anisopliae tanto en estudios in vitro (Cardona et al., 2005; Quinelato et al., 2012) como en su efectividad in vivo para el control de las diferentes fases evolutivas de $\boldsymbol{R}$. microplus en bovinos (Leemon \& Jonsson, 2008), disminuyendo la tasa de ovoposición y eclosión, además de producir la muerte de larvas y garrapatas adultas con porcentajes que oscilan entre el 50 al 100\% (Cardona et al., 2005; Fernández et al., 2010; Rodríguez-Vivas et al., 2011; Meléndez et al., 2012). Diversos estudios revelan efectividad de $\boldsymbol{C}$. bassiana como control biológico sobre poblaciones de garrapatas de la familia Ixodidae, ya sea con aplicación de tratamientos basados únicamente en $\boldsymbol{C}$. bassiana (Ojeda-Chi et al., 2010; Ren et al., 2012), mediante su mezcla con otros productos de origen biológico como M. anisopliae(Kaaya \& Hedimbi, 2012), o en combinación con productos de origen químico (Webster et al., 2015). Algunos acaricidas y pesticidas orgánicos tienen un efecto bioacumulativo en tejidos, como el músculo y la glándula mamaria de los animales expuestos a estos. Al consumir carne y leche, estas sustancias pueden ser biomagnificadas en los seres humanos, exponiendo la salud de los consumidores a mediano y largo plazo (Narváez et al., 2012). La efectividad de los entomopatógenosM. anisopliae y $\boldsymbol{C}$. bassiana ha sido estudiada ampliamente en hatos ganaderos del mundo, considerándolos inocuos para el medio ambiente y la salud humana, tomando gran interés en su uso como acaricidas (Fernández et al., 2010; Pulido-Medellín et al., 2015; García-Corredor et al., 2016).

El objetivo de este estudio fue evaluar in vitro el efecto ixodicida de hongos entomopatógenos (M. anisopliae y $\boldsymbol{C}$. bassiana) y productos químicos (OF, PS y AM) sobre la fase adulta de $\boldsymbol{R}$. microplus, contribuyendo con información para el control de la garrapata en los hatos de la región ganadera en el departamento de Boyacá.

\section{Materiales y Métodos}

El presente estudio se realizó en el Laboratorio de Parasitología Veterinaria de la Universidad Pedagógica y Tecnológica de Colombia (UPTC) en la ciudad de Tunja (Boyacá, Colombia), ubicada en las coordenadas 5³2’7” N, 73²2’04” O. La temperatura promedio anual es de $13^{\circ} \mathrm{C}$, con una altitud de $2782 \mathrm{msnm}$ y una precipitación de $645 \mathrm{~mm}$ al año (Alcaldía de Tunja, 2013). 
Para el estudio se usaron las cepas BbF2011 (C. bassiana) y MAF1309 (M. anisopliae), un OF al 97\%, un PS al 15\% y AM al 20,8\%. Se recolectaron garrapatas hembras adultas pletóricas (0,200-0,250 g) de bovinos del municipio de Moniquirá (Boyacá). Las garrapatas se depositaron en tubos de cristal con tapón de algodón húmedo y fueron trasladadas al Laboratorio de Parasitología Veterinaria de la UPTC para la aplicación de los tratamientos.

Una vez registrado el ingreso de garrapatas al laboratorio, se desinfectaron con cloro (1\%) y se pesaron para crear los grupos a los que se aplicaron los siguientes tratamientos: Grupo I agua destilada, Grupo II C. bassiana $\left(1 \times 10^{8}\right.$ esporas $\left./ \mathrm{ml}\right)$, Grupo III M. anisopliae ( $1 \times 10^{8}$ esporas/ml), Grupo IV OF (concentración de 97\%), Grupo V PS (concentración de 15\%) y Grupo VI AM (concentración de 20,8\%). El primer grupo fue considerado como testigo.

Para evaluar el efecto de los tratamientos sobre la fase adulta de $\boldsymbol{R}$. microplus, su ovoposición (IO) y porcentaje de eclosión (IE), se empleó la técnica de inmersión de hembras repletas durante $60 \mathrm{~s}$ (Drummond \& Whetstone, 1969). El tratamiento se administró individualmente en placas de cultivo de 24 pozos utilizando $1,5 \mathrm{ml}$ del biopreparado o $1,5 \mathrm{ml}$ del producto químico. Una vez fuera de los pozos, las garrapatas se secaron e incubaron durante 21 días en cámara húmeda $(85 \% \pm 1$ de humedad relativa) a $28^{\circ} \mathrm{C} \pm 1$. Se realizaron observaciones diarias al microestereoscopio para comprobar el desarrollo de micosis o el efecto del fármaco.

Para determinar el efecto de los tratamientos sobre la ovoposición de $\boldsymbol{R}$. microplus, se retiró la postura de cada garrapata a los 14 días de incubación, luego se pesaron e incubaron los huevos bajo condiciones controladas de humedad y temperatura $(85 \% \pm 1$ HR y $28^{\circ} \mathrm{C} \pm 1$ ). Para realizar los cálculos del efecto de cada tratamiento sobre la ovoposición se obtuvo la relación del peso de huevos depositados / peso de las hembras por grupo y lote (Álvarez et al., 2008):

$$
O P=\frac{(\text { Pesodehevos en gramos }) x(20000)}{\text { Pesodehembras en gramos }} \times(100)
$$

Una vez obtenido este parámetro, tanto para individuos tratados como para los del grupo testigo, se aplicó la siguiente fórmula (Bravo et al., 2008):

$$
\% 1 O=\frac{(O P / T-O P / t) x(100)}{(O P / T)}
$$

Donde:

OP/T = Reproducción estimada del grupo testigo.

$\mathrm{OP} / \mathrm{t}=$ Reproducción estimada del grupo tratado.

Para calcular la eclosión de larvas, se tomaron cinco alícuotas y se realizó el conteo de cascarones rotos y no rotos hasta completar 100 huevos contados procedentes de una muestra de $1 \mathrm{~g}$ de huevos incubada a $28^{\circ} \mathrm{C}$ y $85 \%$ HR por 35 días y previamente homogenizada. Con los resultados de los conteos se obtuvo el promedio de eclosión para los diferentes tratamientos. 
El porcentaje de eclosión se estableció por medio de la siguiente fórmula:

$$
\% \mathrm{E}=\mathrm{C} / \mathrm{H}+\mathrm{C} \times 100
$$

$\mathrm{C}=$ Cascarones, $\mathrm{H}=$ Huevos.

Se calculó la eficiencia reproductiva (ER), según cada acaricida y el control, La ER es un valor necesario para calcular la eficacia del acaricida (Drummond \& Whetstone, 1969):

$$
\mathrm{ER}=\frac{\text { Peso de los huevos }}{\text { Peso de las garrapatas }} \quad \mathrm{x} \% \text { de Eclosión x } 20.000
$$

Se calculó la eficacia del producto (EP), según cada acaricida (Abbott, 1925):

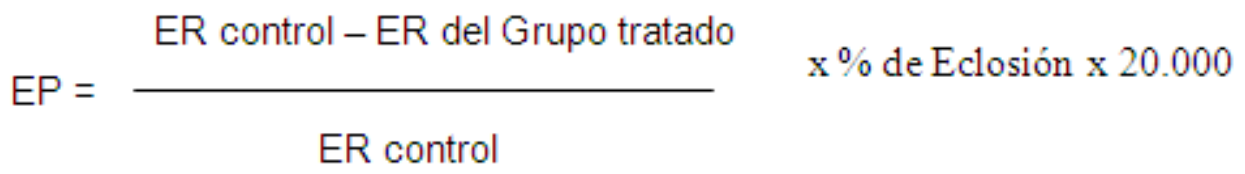

El factor de resistencia se calculó dividiendo la eficacia esperada 100\% entre la eficacia obtenida en cada producto.

Se utilizó un diseño experimental completamente al azar con seis tratamientos y tres repeticiones, cada repetición con 10 garrapatas por tratamiento. Los datos fueron analizados en el programa estadístico EPIDAT 4.0.

\section{Resultados y Discusión}

El tratamiento que ocasionó la primera muerte en menor tiempo fue AM (3 días PT), seguido por PS (4 días PT); para el OF, C. bassiana y M. anisopliae la primera muerte se registró el día 6 PT; para el tratamiento control se observó la primera muerte al día 17 PT. Los días promedio para el 50\% de muertes se alcanzaron en menor tiempo con OF a los 6 días PT, seguido de $\boldsymbol{M}$. anisopliae con 7 días PT y $\boldsymbol{C}$. bassiana 8 días PT; para los tratamientos AM, PS y control el 50\% de la mortalidad se alcanzó a los 12, 19 y 20 días PT, respectivamente. El $100 \%$ de mortalidad en las garrapatas fue logrado por el tratamiento con $\boldsymbol{C}$. bassiana a los 10 días PT, con $\boldsymbol{M}$. anisopliae se observó a los 11 días PT (Tabla 1). En estudios previos se ha demostrado que los hongos entomopatógenos a concentraciones de 1 x108 esporas/ml son altamente efectivos para el control de $\boldsymbol{R}$. microplus alcanzando mortalidades entre 44 al 100\% (Ojeda-Chi et al., 2010; Fernandes et al., 2012; Ren et al., 2012; Sônia et al., 2012; Pulido-Medellín et al., 2015; García-Corredor et al., 2016).

Para el caso de OF a los 14 días se logró el 100\% de mortalidad, valor equivalente al trabajo realizado por Céspedes et al. (2002) donde se alcanzaron mortalidades de 88,3 a 
92,8\% en garrapatas $\boldsymbol{R}$. microplus. Por el contrario, resultados de baja mortalidad fueron encontrados por Araque et al. (2014), observándose 13\% de mortalidad en garrapatas adultas de la misma especie estudiada; los tratamientos con AM, PS y control no alcanzaron el $100 \%$ de la mortalidad en las garrapatas en el tiempo determinado para el desarrollo del trabajo (21 días); AM alcanzo un 60\% de mortalidad, resultado comparable con porcentajes encontrados en 3 regiones de Perú con mortalidades de $85 \%$ en las garrapatas de la provincia Cutervo y 28,10\% en la provincia Coronel Portillo, considerándose resistentes a todos aquellos productos que no alcanzaron el 95\% de eficacia (Rojas \& Portal, 2013).

El tratamiento con PS obtuvo una mortalidad de 56,6\%, González et al. (2011) evaluaron 4 tipos de ixodicidas incluyendo un piretroide con efectividad del 40,9\%. En México identificaron poblaciones de garrapatas multirresistentes a compuestos organofosforados, piretroides sintéticos, amitraz y lactonas macrocíclicas (FernándezSalas et al., 2012). En un estudio realizado en cinco estados de Brasil, se reportó la existencia de poblaciones de $\boldsymbol{R}$. microplus resistentes a los piretroides sintéticos, al amitraz y a los organofosforados, con frecuencias del 94, 88 y 82\%, respectivamente (Lovis et al., 2013).

En todos los tratamientos se calculó el porcentaje de inhibición de la ovoposición (Figura 1). El tratamiento que logró mayor eficiencia fue OF (98\%), seguido por los hongos entomopatógenos $\boldsymbol{M}$. anisopliae (80,7\%) y $\boldsymbol{C}$. bassiana (75\%). AM y PS obtuvieron un porcentaje de inhibición más bajo con $60 \%$ y 59,7\%, respectivamente.

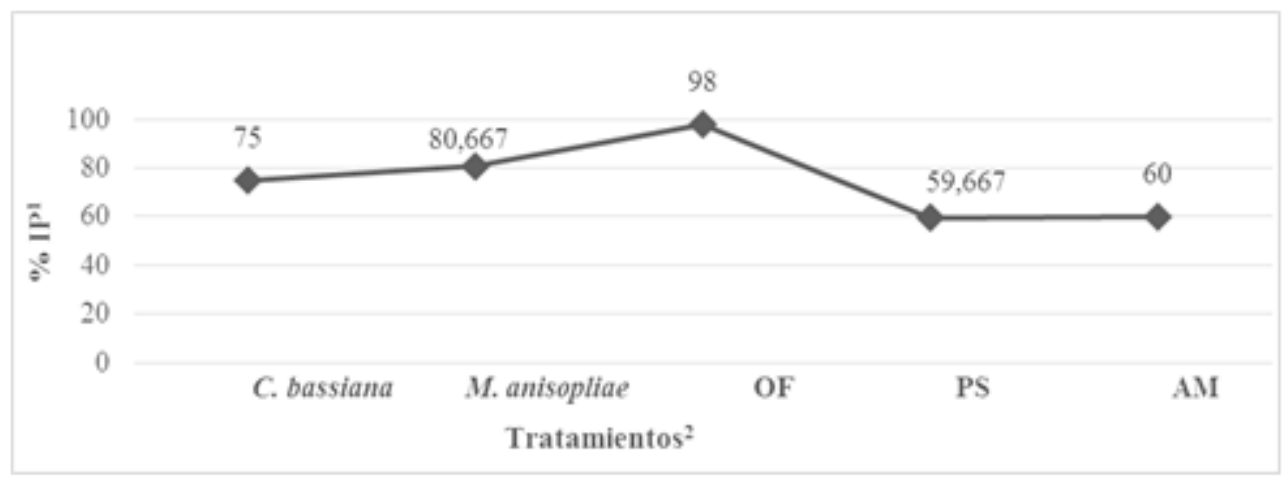

${ }^{1} \%$ IP porcentaje ovoposición. ${ }^{2}$ OF: Organofosforado. AM: Amitraz. PS: Piretroide sintético.

Figura 1. Efecto de cada tratamiento sobre el porcentaje de ovoposición (\%IP) en garrapatas adultas R. microplus.

En cuanto al porcentaje de eclosión de huevos, el tratamiento que mostró mayor efecto inhibitorio fue OF con 70,3\%, seguido por $\boldsymbol{M}$. anisopliae (65\%) у C. bassiana(63,3\%). Los tratamientos que menos afectaron la eclosión de los huevos fueron AM y PS con 3,7\% (Figura 2). Ren et al. (2012) determinaron la acción patogénica de $\boldsymbol{M}$. anisopliae (MaAT04) al disminuir el índice de eficiencia reproductiva de $\boldsymbol{R}$. microplusy al causar una mortalidad del 100\% en hembras ingurgitadas a la concentración de 108 y 109 conidios/ml, 15 días después de aplicado el tratamiento. 


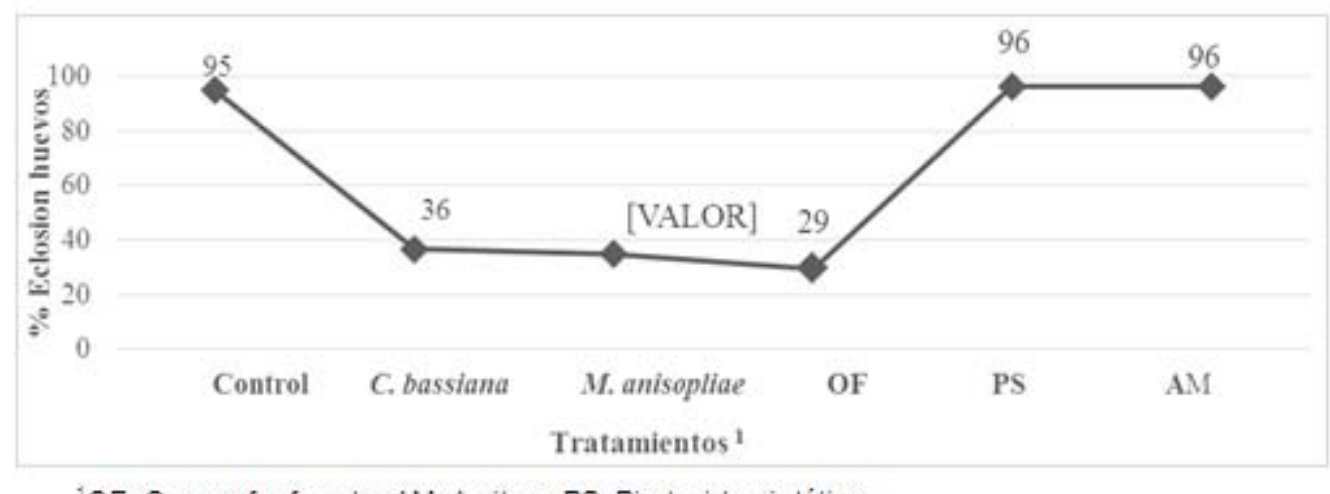

OF: Organofosforado. AM: Amitraz. PS: Piretroide sintético.

Figura 2. Porcentaje de huevos eclosionados por efecto de cada tratamiento en garrapatas adultas de $R$. microplus.

En la evaluación de los parámetros reproductivos se encontró que los tratamientos con OF, $\boldsymbol{M}$. anisopliae y $\boldsymbol{C}$. bassiana disminuyeron significativamente la reproducción estimada: $81,3 \%$, 80,9\% y 75,2\%, respectivamente. Pulido-Medellín et al. (2015) observaron que las garrapatas tratadas con la cepa MaF1309 de $\boldsymbol{M}$. anisopliaereducen en 91\% el índice de eficiencia reproductiva, sugiriendo que los hongos entomopatógenos a mayor concentración generan mayor efecto patógeno (Fernández et al., 2010; Ren et al., 2012; Valverde-Sánchez et al., 2015). En contraposición, se ha reportado que el hongo entomopatógeno $\boldsymbol{M}$. anisopliae secreta enzimas como proteasas, quitinasas, lipasas y esterasas encargadas de la degradación de la cutícula de los artrópodos (Sandhu et al., 2012). En consecuencia, Sapna et al. (2012) demostraron que la virulencia de aislados de $\boldsymbol{M}$. anisopliae es proporcional al incremento de la cantidad de enzimas producidas por el hongo independiente de la concentración.

El \%IP aumentó en todos los tratamientos teniendo resultados notables para el tratamiento con OF (98\%), M. anisopliae (80,7\%) y C. bassiana (75\%). Perinotto et al. (2012) observaron que en los huevos tratados con M. anisopliae disminuyó entre el 50,5 y 57,3\% la eclosión, con un aislamiento de la cepa Ma959 y una concentración de $1 \times 10^{8}$ conidias $/ \mathrm{ml}$. Al tratarse de métodos de control biológico, los resultados encontrados para controlar el potencial reproductivo fueron aceptables, teniendo en cuenta que la prueba utiliza garrapatas repletas, estadio en que son más tolerantes, por lo menos al control químico (Ming et al., 2013); así lo demostraron López et al. (2009), donde la efectividad de un producto (mezcla de un PS con un OF) sobre la ovoposición y fertilidad de los huevos fue del 100\%. La eclosión de los huevos se vio afectada por cada uno de los tratamientos empleados en este trabajo con porcentajes de $29,7 \%$ para OF, 35\% para $\boldsymbol{M}$. anisopliae y 36,7\% para $\boldsymbol{C}$. bassiana, comparados con resultados de 85\% a 100\% con cepas Fitossan 3 de $\boldsymbol{C}$. bassiana donde aumentaron la concentración del hongo a 1x109 conidias/ml (Sônia et al., 2012); esto podría sugerir que las concentraciones para los hongos empleados en este trabajo afectaron la ovoposición y la eclosión de huevos de $\boldsymbol{R}$. microplus, lo que no permitió la emergencia de larvas infestantes de manera normal, obteniendo un control aceptable sobre estos parámetros.

Para determinar la eficacia de los productos se calculó la eficiencia reproductiva (ER), valor que expresa la capacidad de una teleogina para transformar su peso corporal en larvas viables (Bravo, 2008). En cuanto a la eficacia de los productos, PS y AM mostraron valores inferiores a la eficacia mínima aceptada internacionalmente (Tabla 
2), la cual debe ser igual o mayor de 90\% (FAO, 2004). Los tratamientos con $C$. bassianay $\boldsymbol{M}$. anisopliae tuvieron una eficacia de 98 y 99\% respectivamente con un factor de resistencia de 1,02 y 1,01 demostrando la efectividad de estos productos.

Es importante destacar el potencial que presentan los hongos entomopatógenos en el control de garrapatas $\boldsymbol{R}$. microplus, constituyéndolos en una de las alternativas dentro de las explotaciones bovinas, ya sea solos o en combinación mediante aplicaciones acuosas repetitivas como lo afirman Rodríguez-Alcocer et al. (2014), quienes resaltan la utilización de dos cepas de $\boldsymbol{M}$. anisopliae en mezcla (Ma14 + Ma34) a la concentración 1 x 108 conidias/ml sobre bovinos, reduciendo la población de $\boldsymbol{R}$. microplus desde la primera aplicación, controlando la garrapata en su estado larvario, ninfal y adulto. Webster et al. (2015) realizaron aplicaciones de conidias de $\boldsymbol{M}$. anisopliae (108 conidios/ml), solución acaricida de cipermetrina más clorpirifos, y la mezcla del hongo con la solución acaricida sobre ganado infestado con hembras repletas de $\boldsymbol{R}$. microplus, los resultados revelaron una eficacia media de 56,3, 71,1 y 97,9\%, respectivamente.

Tabla 1. Eficacia (\%) de productos para el control de garrapatas adultas $R$. microplus según el día postratamiento

\begin{tabular}{cccccccc}
\hline Tratamiento'/Dias & 3 & 6 & 9 & $\mathbf{1 2}$ & $\mathbf{1 5}$ & $\mathbf{1 8}$ & $\mathbf{2 1}$ \\
\hline Control & 0 & 0 & 0 & 0 & 0 & 4,3 & 4,3 \\
\hline C. bassiana & 0 & 3 & 7,6 & 10 & 10 & 10 & 10 \\
\hline M. anisopliae & 0 & 1,3 & 7,3 & 10 & 10 & 10 & 10 \\
\hline OF & 0 & 6 & 7 & 9,3 & 9,3 & 9,3 & 10 \\
\hline AM & 2,6 & 3,6 & 4 & 4,6 & 5 & 6 & 6 \\
\hline PS & 0 & 2,7 & 3 & 3 & 3,3 & 5,6 & 5,6 \\
\hline OF: Organofosforado. AM: Amitraz. PS: Piretroide sintético. & & & &
\end{tabular}

Tabla 2. Eficacia y factor de resistencia según tratamiento

\begin{tabular}{|c|c|c|}
\hline Tratamientos $^{1}$ & Eficacia & Factor de resistencia \\
\hline AM & $64 \%$ & 1,65 \\
\hline PS & $57,76 \%$ & 1,75 \\
\hline OF & $98 \%$ & 1,02 \\
\hline M. anisopliae & $98 \%$ & 1,02 \\
\hline C. bassiana & $99 \%$ & 1,01 \\
\hline
\end{tabular}

\section{Conclusiones}

Los resultados encontrados para el control de garrapatas adultas de $\boldsymbol{R}$. microplus en condiciones de laboratorio fueron favorables para los tratamientos con $\boldsymbol{M}$. anisopliae yC. bassiana, comprobando que estos hongos entomopatógenos no solo causan la mortalidad en la fase adulta de las garrapatas, sino que además tienen gran eficacia sobre su reproducción. Los buenos resultados que mostró el tratamiento con OF permiten concluir que aún no hay resistencia de $\boldsymbol{R}$. microplus a este compuesto químico, por tanto, es recomendable la realización de otros experimentos utilizando el 
control integrado con el uso de productos químicos y hongos entomopatógenos, o asociaciones de productos biológicos ya que estos no producen efectos adversos al medio ambiente (Narváez et al., 2012). Las cepas BbF2011 del hongo C. bassiana y MAF1309 del hongo $M$. anisopliae y el OF al 97\% de concentración presentan los mejores resultados en la eficiencia in vitro en el control de garrapatas adultas, reduciendo los índices de eclosión de huevos, ovoposición y aumentado los niveles de mortalidad de $\boldsymbol{R}$. microplus.

\section{Referencias bibliográficas}

- Abbott, W. A method of computing the effectiveness of an insecticide. Journal of Economic Entomology, v18, p. 256-257, 1925.

- Alcaldía de Tunja. Nuestro Municipio; 2013. Disponible en: Link

- Álvarez, V.; Loaiza, J.; Bonilla, R.; Barrios, M. Control in vitro de garrapatas (Boophilus microplus; Acari: Ixodidae) mediante extractos vegetales. Revista de Biología Tropical, v.56, n.1, p.291-302, 2008.

- Antunes, S.; Merino, O.; Lérias, J.; Domingues, N.; Mosqueda, J.; de la Fuente, J.; Domingos, A. Artificial feeding of Rhipicephalus microplus female ticks with anti calreticulin serum do not influence tick and Babesia bigemina acquisition. Ticks and Tick-borne Diseases, v.6, n.1, p.47-55, 2015.

- Araque, A.; Ujueta, S.; Bonilla, R.; Gómez, D.; Rivera, J. Resistencia a acaricidas en Rhipicephalus (Boophilus) microplus de algunas explotaciones ganaderas de Colombia. Revista U.D.C.A. Actualidad \& Divulgación Científica, v.17, n.1, p.161170, 2014.

- Brahma, R.K.; Dixit, V.; Sangwan, A.K.; Doley, R. Identification and characterization of Rhipicephalus (Boophilus) microplus and Haemaphysalis bispinosa ticks (Acari: Ixodidae) of North East India by ITS2 and 16S rDNA sequences and morphological analysis. Experimental and Applied Acarology, v.62, p.253-265, 2014.

- Bravo, M.; Coronado, A.; Henríquez, H. Eficacia in vitro del amitraz sobre poblaciones Boophilus microplus provenientes de explotaciones lecheras del estado Lara, Venezuela. Zootecnia Tropical, v.26, n.1, p.35-40, 2008.

- Cardona, E.; Montoya, M.; Arias, A. Evaluación in vitro de Metarhizium anisopliae y Beauveria bassiana sobre hembras ingurgitadas de Dermacentor nitens (Acari:Ixodidae). Universidad de Antioquia. Revista Colombiana de Ciencias Pecuarias, v.4, n.18, p.384, 2005. 
- Céspedes, N.; Santamaría, M.; Frogoso, H.; García, Z. Primer caso de resistencia al AM en la garrapata del ganado Boophilus microplus. Técnica Pecuaria en México, v.40, n.1, p.81-92, 2002.

- Chagas, A.C.S.; Domingues, L.F.; Fantatto, R.R.; Giglioti, R.; Oliveira, M.C.S.; Oliveira, D.H.; Mano, R.A.; Jacob, R.G. In vitro and in vivo acaricide action of juvenoid analogs produced from the chemical modification of Cymbopogon spp. and Corymbia citriodora essential oil on the cattle tick Rhipicephalus (Boophilus) microplus. Veterinary Parasitology, v.205, n-1-2, p.277-284, 2014.

- Cortes-Vecino, J.A.; Betancourt-Echeverri, J.A.; Argüelles-Cárdenas, J.; PulidoHerrera, L.A. Distribución de garrapatas Rhipicephalus (Boophilus) microplus en bovinos y fincas del Altiplano Cundiboyacense (Colombia). Corpoica Ciencia y Tecnología Agropecuaria, v.11, n.1, p.73-84, 2010.

- Cruz-Vázquez, C.; Cruz-Avalos, A.; Lezama-Gutiérrez, R.; Angel-Sahagún, C.; VitelaMendoza, I. Selección de aislados de hongos entomopatógenos para el control de Rhiphicephalus microplus (Acari: Ixodidae). Tropical and Subtropical Agroecosystems, v.18, p.175-180, 2015.

- De Clercq, E.M.; Leta, S.; Estrada-Peña, A.; Madder, M.; Adehan, S.; Vanwambeke, S.O. Species distribution modelling for Rhipicephalus microplus(Acari: Ixodidae) in Benin, West Africa: Comparing datasets and modelling algorithms.Preventive Veterinary Medicine, v.118, n.1, 8-21, 2015.

- Drummond, O.; Whetstone, M. Oviposition of the gulf coast tick. Journal of Economic Entomology, v.63, n.5, p.1547-1551, 1969.

- Food and Agriculture Organzaition of the United Nations, [FAO]. Guidelines for Resistance Management and Integrated Parasite Control in Ruminants. 2004.

- FAO. Unión Ganadera Regional de Jalisco; 2013. Generado: 23 de febrero, Recuperado de: Link

- Fernandes, E.; Bittencourt, E.; Roberts, D. Perspectives on the potential of entomopathogenic fungi in biological control of ticks. Experimental Parasitology, v.130, n.3, p.300-305, 2012.

- Fernández, M.; Berlanga, A.; Cruz, C.; Hernández, V. Evaluación de cepas de Beauveria bassiana y Metarhizium anisopliae sobre la inhibición de oviposición, eclosión y potencial reproductivo en una cepa triple resistente de garrapata Rhipicephalus (Boophilus) microplus (Canestrini) (Acari: Ixodidae). Revista de la Sociedad Venezolana de Entomología, v.25, n.3, p.109-115, 2010. 
- Fernández-Salas, A.; Rodríguez-Vivas, R.I.; Alonso-Díaz, M.A. First report of aRhipicephalus microplus tick population multi-resistant to acaricides and ivermectin in the Mexican tropics. Veterinay Parasitology, v.98, n.5, p.1010-1014, 2012.

- Galay, R.L.; Umemiya-Shirafuji, R.; Mochizuki, M.; Fujisaki, K.; Tanaka, T. Iron metabolism in hard ticks (Acari: Ixodidae): The antidote to their toxic diet.Parasitology International, v.64, n.2, p.182-189, 2015.

- García-Corredor, D.J.; Rodríguez-Vivas, R.; Pulido-Medellín, M.; Andrade-Becerra, R.; Díaz-Anaya, A. Evaluación in vitro de Cordyceps bassiana (Ascomycota: Sordariomycetes) en el Control Biológico de Rhipicephalus microplus. Revista de Investigaciones Veterinarias del Perú, v.27, n.1, p.130-136, 2016.

- González, A.; Tapias, D.; Pérez, M.; Carvajalino, M.; Velandia, D.; Borges R. Evaluación de Acaricidas para el control de garrapatas (Rhipicephalus (Boophilus) microplus) que afectan al ganado bovino de doble propósito usando modelos lineales generalizados. Revista de la Facultad de Agronomía de la Universidad del Zulia, v.28, n.4, p.487-502, 2011.

- Kaaya, G.; Hedimbi, M. The use of entomopathogenic fungi, Beauveria bassiana andMetharizium anisopliae, as bio-pesticides for tick control. International Journal of Agriculture Sciences Abstracted, v.2, n.6, p.245-250, 2012.

- Leemon, D.M.; Jonsson, N.N. (2008). Laboratory studies on Australian isolates of Metarhizium anisopliae as a biopesticide for the cattle tick Boophilus microplus.Journal of Invertebrate Pathology, v.97, n.1, p.40-49, 2008.

- López, G.; Grisi, C.; Gómez, J.; Valencia, L.; González, D. Evaluación de una mezcla de cipermetrina + clorpirifós sobre la garrapata Rhipicephalus (Boophilus) microplus en pruebas de campo y de laboratorio en el predio Esteban Jaramillo Román Gómez del Politécnico Colombiano de Marinilla, Antioquia. Revista CES, Medicina Veterinaria y Zootecnia, v.4, n.2, p.57-65, 2009.

- Lovis, L.; Mendes, M.C.; Perret, J.-L.; Martins, J.R.; Bouvier, J.; Betschart, B.; Sager, H. Use of the Larval Tarsal Test to determine acaricide resistance in Rhipicephalus (Boophilus) microplus. Brazilian field populations. Veterinay Parasitology, v.191, n.34, p.323-31, 2013.

- Meléndez, B.; Trejo, N.; Trabanino, R. Evaluación de Beauveria bassiana y Metarhizium anisopliae para el control de Rhipicephalus (Boophilus) microplus en vacas del hato lechero de Zamorano. Escuela Agrícola Panamericana; 2012. 
- Ming, S.; Qiaoyun, R.; Guiquan, G.; Yufeng, L.; Xueqing, H.; Chao, M.; Hong, Y.; Jianxun L. Effectiveness of Beauveria bassiana sensu lato strains for biological control against Rhipicephalus (Boophilus) microplus (Acari: Ixodidae) in China. Parasitology International, v.62, n.20, p.412-415, 2013.

- Monteiro, C.M.O.; Araújo, L.X.; Gomes, G.A.; Senra, T.O.S.; Calmon, F.; Daemon, E.; de Carvalho, M.G.; Bittencourt, V.R.E.P.; Furlong, J.; Prata, M.C. A. Entomopathogenic nematodes associated with essential oil of Lippia sidoides for control of Rhipicephalus microplus (Acari: Ixodidae). Parasitology Research, v.113, n.1, p.189-195, 2014.

- Narváez, J.F.; Palacio, J.A.; Molina, F.J. Persistencia de plaguicidas en el ambiente y su ecotoxicidad: una revisión de los procesos de degradación natural. Gestión y Ambiente, v.15, n.3, p.27-38, 2012.

- Ojeda-Chi, M.M.; Rodríguez-Vivas, R.I.; Galindo-Velasco, E.; Lezama-Gutiérrez, R. Laboratory and field evaluation of Metarhizium anisopliae (Deuteromycotina: Hyphomycetes) for the control of Rhipicephalus microplus (Acari: Ixodidae) in the Mexican tropics. Veterinary Parasitology, v.170, n.3-4, p.348-354, 2010.

- Ortiz-Urquiza, A.; Luo, Z.; Keyhani, N.O. Improving mycoinsecticides for insect biological control. Applied Microbiology and Biotechnology, v.99, n.3, p.1057-1068, 2015.

- Perinotto, W.M.S.; Angelo, I.C.; Gôlo, P.S.; Quinelato, S.; Camargo, M.G.; Sá, F.; Bittencourt, R.E.P. Susceptibility of different populations of ticks to entomopathogenic fungi. Experimental Parasitology, v.130, n.3, p.257-260, 2012.

- Pulido-Medellín M, Rodríguez-Vivas R, García-Corredor D, Díaz-Anaya A, AndradeBecerra R. Evaluación de la Eficacia de la Cepa MaF1309® deMetarhizium anisopliae en el Control Biológico de Garrapatas Adultas deRhipicephalus microplus en Tunja, Colombia. Revista de la Facultad-Universidad Central de Venezuela, v.56, n.2, p.75-81, 2015.

- Quinelato, S.; Gôlo, P.S.; Perinotto, W.M.S.; Sá, F.A.; Camargo, M.G.; Angelo, I.C.; Moraes, A.M.L.; Bittencourt, V.R.E.P. Virulence potential of Metarhizium anisopliae s.l. isolates on Rhipicephalus (Boophilus) microplus larvae. Veterinay Parasitology, v.190, n.3-4, p.556-65, 2012.

- $\quad$ Ren, Q.; Liu, Z.; Guan, G.; Sun, M.; Ma, M.; Niu, Q.; Li, Y.; Liu, A.; Liu, J.; Yang, J.; Yin, H.; Luo, J. Laboratory evaluation of virulence of Chinese Beauveria 
bassiana and Metarhizium anisopliae isolates to engorged female Rhipicephalus (Boophilus) microplus ticks. Biological Control, v.63, n.2, p.98-101, 2012.

- Rodríguez-Alcocer, U.J.; Rodríguez-Vivas, R.I.; Ojeda-Chi, M.M.; Galindo-Velasco, E.; Lezama-Gutiérrez, R. Efficacy the mixture of two strains of Metarhizium anisopliae (Deuteromycotina: Hyphomycetes) to control Rhipicephalus microplus on natural infestation of cattle. Tropical and Subtropical Agroecosystems, v.17, n.2, p.223-229, 2014.

- Rodríguez-Vivas, R.; Arieta-Román, J.; Pérez-Cogollo, L.; Rosado-Aguilar, J.; Ramírez-Cruz, G.; Basto-Estrella, G. Uso de lactonas macrocíclicas para el control de la garrapata Rhipicephalus (Boophilus) microplus en el ganado bovino. Archivos de Medicina Veterinaria, v.42, n.3, p.115-123, 2010.

- Rodríguez-Vivas, R.; Torres, J.; Ramírez, G.; Rosado-Aguilar, A.; Aguilar-Caballero, J.; Ojeda-Chi, M.; Bolio, M. Manual técnico: Control de parásitos internos y externos que afectan al Ganado bovino en Yucatán, México. Resistencia de Rhipicephalus (Boophilus) microplus y nematodes gastrointestinales a la ivermectina en ranchos bovines de Yucatán, v.52, 2011. UADY. CONACYT.

- Rodríguez-Vivas, R.I.; Hodgkinson, J.E.; Trees, A.J. Resistencia a los acaricidas en Rhipicephalus (Boophilus) microplus: situación actual y mecanismos de resistencia.Revista Mexicana de Ciencias Pecuarias, v.3, supl.1, p.9-24, 2012.

- Rojas, J.; Portal, J. Detección in vitro de resistencia de garrapatas Boophilus microplus a deltametrina 12.5\%, AM 5\% y coumaphos 50\% en tres provincias de Perú. Sitio Argentino de Producción Animal, p.1-4, 2013.

- Sandhu, S.S.; Sharma, A.K.; Beniwal, V.; Goel, G.; Batra, P.; Kumar, A.; Jaglan, S.; Sharma, A.K.; Malhotra, S. Myco-biocontrol of insect pests: factors involved, mechanism, and regulation. Journal of Pathogens, 2012. doi:10.1155/2012/126819.

- Sapna, N.B.; Remadevi, O.K.; Sasidharan, T.O.; Balachander, M.; Priyadarsanan Dharmarajan. Cuticle degrading enzyme production by some isolates of the entomopathogenic fungus, Metarhizium anisopliae (Metsch.). Journal of Biosciences, v.20, p.25-32, 2012.

- Sônia, F.; Broglio-Micheletti, L.; De-Souza, E.; Valente, M.; De-Araújo, J.; Da-Silva, N.; Gómez-Torres, M. Evaluation of entomopathogenic fungi as biological control agents Rhipicephalus $\quad$ (Boophilus) microplus (Canestrini, 1887 ) (Acari: Ixodidae). Idesia (Arica), v.30, n.1, p.93-99, 2012. 
- Valverde-Sánchez, A.L.; Mata-Granados, X.; Lezama-Gutiérrez, R.; Camacho-Calvo, M. Metarhizium anisopliae como mico-acaricida para el control de la garrapataRhipicephalus microplus. Revista Iberoamericana de Ciencias, v.6, n.2, p.1-18, 2015.

- Webster, A.; Reck, J.; Santi, L.; Souza, U.A.; Dall’Agnol, B.; Klafke, G.M.; Beys-daSilva, W.O.; Martins, J.R.; Schrank, A. Integrated control of an acaricide-resistant strain of the cattle tick Rhipicephalus microplus by applying Metarhizium anisopliae associated with cypermethrin and chlorpyriphos under field conditions. Veterinary Parasitology, v.207, n.3-4, p.302-308, 2015.

Cómo citar: Sepúlveda, A.L.; Pulido-Medellín, M.O.; Rodríguez-Pacheco, J.E.; GarcíaCorredor, D.J. Eficiencia in vitro de hongos entomopatógenos y productos químicos sobre Rhipicephalus microplus. Revista Veterinaria y Zootecnia, v.11, n.2, p.67-80, 2017. DOI: 10.17151/vetzo.2017.11.2.6

Esta obra está bajo una Licencia de Creative Commons Reconocimiento CC BY

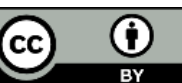

